\title{
Erkrankungen der Fesselbeugesehnenscheide - eine diagnostische und therapeutische Herausforderung
}

\author{
Carolin Gerdes
}

Die Erkrankungen der Fesselbeugesehnenscheide stellen im Praxisalltag immer wieder eine Herausforderung dar. Der folgende Beitrag beschäftigt sich mit der Diagnosestellung und der daraus resultierenden Therapie bei Lahmheiten, deren Ursache auf den Bereich der Fesselbeugesehnenscheide lokalisiert ist.

\section{Lokalisierung der Lahmheitsursache}

Die Lokalisierung der Lahmheitsursache auf die Fesselbeugesehnenscheide erfolgt durch die intrasynoviale Anästhesie. Bei der Interpretation der Ergebnisse sollte man beachten, dass auch die palmaren/ plantaren digitalen Nerven dadurch desensibilisiert werden können [14]. Eine Sensibilitätsprobe am Hufballen oder eine vorherige, separate Ausschaltung der Nerven distal der Sehnenscheide mittels Leitungsanästhesie schließt falsch positive Ergebnisse aus. Ein positives Ergebnis der intrasynovialen Anästhesie ist dann höchstwahrscheinlich auf Schmerzen im Bereich der Sehnenscheide zurückzuführen [13].

Manchmal wird mit der intrasynovialen Anästhesie nur eine partielle Verbesserung der Lahmheit erreicht. In diesen Fällen sollte eine Leitungsanästhesie durchgeführt werden. Die tiefe Vierpunktanästhesie (mittlere palmare/plantare Anästhesie, MPA) resultiert in positiven Fällen in einer signifikanten Verbesserung der Lahmheit. Bei einem positiven Ergebnis der tiefen Vierpunktanästhesie sollten Lahmheiten der Zehe und des Fesselgelenks mithilfe einer tiefen palmaren/plantaren Anästhesie (TPA 1 oder 2) und einer intrasynovialen Anästhesie des Fesselgelenks separat ausgeschlossen werden.

\section{Weiterführende Untersuchungen}

Wurde die Lahmheitsursache auf den Bereich der Fesselbeugesehnenscheide lokalisiert, können folgende weiterführende Untersuchungen vorgenommen werden:

- röntgenologische Untersuchung

- röntgenologische Kontrastmitteluntersuchung [9]

- sonografische Untersuchung

- diagnostische Tendovaginoskopie
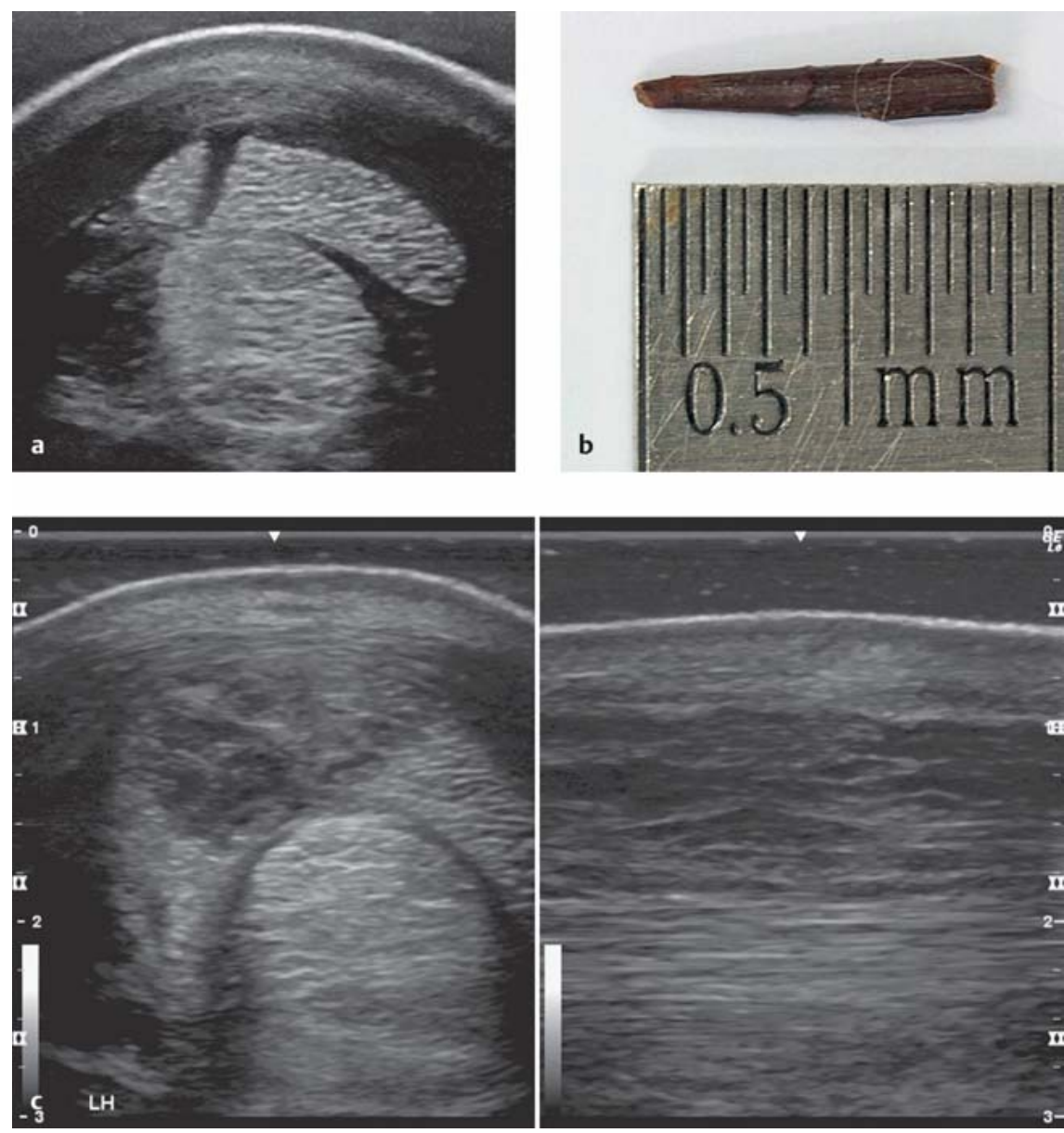

Abb. 1 a Transversales Ultraschallbild Zone 3B: Läsion der oberflächlichen Beugesehne durch Trauma. b Fremdkörper nach chirurgischer Entfernung. Dornen können in die Sehnenscheide penetrieren, verschiedene Strukturen verletzen und zu einer septischen Sehnenscheidenentzündung führen. Bei der klinischen Untersuchung ist die Penetrationsstelle häufig unauffällig. c Transversales (links) und longitudinales (rechts) Ultraschallbild Zone 3B: septische Tendinitis der oberflächlichen Beugesehne nach traumatischer Verletzung. (c) C. Gerdes

Die röntgenologische Untersuchung des Fesselgelenks und der proximalen Zehe sollte routinemäßig durchgeführt werden und ist besonders wichtig bei undeutlichen Ergebnissen der diagnostischen Anästhesie. In diesen Fällen könnte die Lahmheit andere Ursachen haben (z. B. eine Arthrose des Fesselgelenks oder Veränderungen der proximalen Sesambeine), die eventuell von einer Sehnenscheidenentzündung begleitet werden.

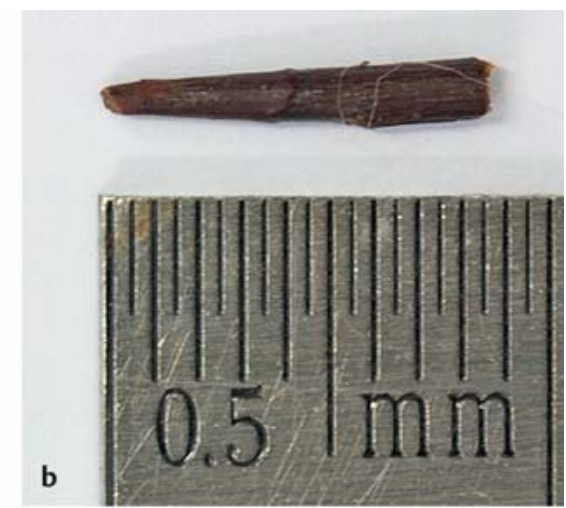



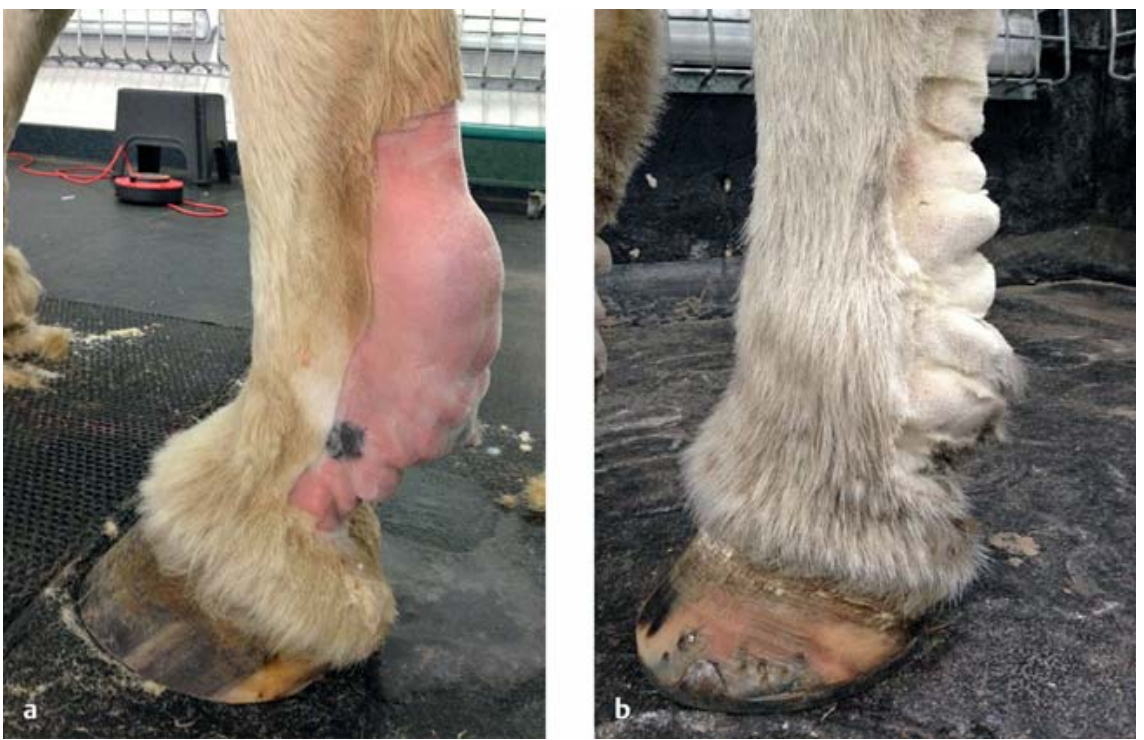

Abb. 2 a Vermehrte Füllung der Fesselbeugesehnenscheide nach dem Scheren. b Schwellung im Bereich der Fesselbeugesehnenscheide. Für manche schwere Rassen (z. B. Cobs) sind diese verdickten Hautfalten häufig, was die klinische und ultrasonografische Beurteilung der Fesselbeugesehnenscheide unmöglich macht. Leider sind gerade schwere Rassen besonders häufig von Entzündungen der Fesselbeugesehnenscheide betroffen. (c) C. Gerdes

\section{Differenzialdiagnosen}

Bei Lokalisierung der Lahmheitsursache auf den Bereich der Fesselbeugesehnenschneide kommen folgende Differenzialdiagnosen infrage:

- septische Tendovaginitis

- aseptische primäre Tendovaginitis

- aseptische sekundäre Tendovaginitis

\section{Septische Tendovaginitis}

Eine septische Tendovaginitis kann aufgrund einer perforierenden Verletzung ( $\triangleright$ Abb. 1), infolge einer hämatogenen Verbreitung der Erreger oder iatrogen entstehen.

Bei einer septischen Tendovaginitis ist die Zahl der Leukozyten in der Synovialflüssigkeit erhöht. Bei einer Erhöhung der Leuko-

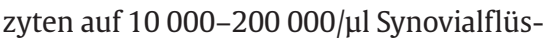
sigkeit wird von einer septischen Infektion gesprochen. Wenn die Ursache der Infektion eine perforierende Verletzung der Fesselbeugesehnenscheide ist, sollte immer auf zusätzliche Sehnen-, Band- und Knochenverletzungen untersucht werden, da diese einen negativen Einfluss auf die Prognose haben [20]. Die Behandlung erfolgt immer chirurgisch. Bei den betroffenen Pferden sollte schnellstmöglich eine Lavage der Sehnenscheide durchgeführt werden. Diese erfolgt entweder beim ste- henden Pferd unter Sedierung oder vorzugsweise im Rahmen einer Tendovaginoskopie unter Vollnarkose. Alternativ kann das Pferd umgehend in eine entsprechend ausgestattete Klinik überwiesen werden.

\section{.ps}

Die Zeit zwischen dem Auftreten der klinischen Symptome und der chirurgischen Behandlung hat einen deutlichen Einfluss auf die Prognose [19].

Eine stark vermehrte Füllung der Sehnenscheide - wie z.B. bei einer septischen Tendosynovitis - führt in der Regel zu einem erhöhten Druck auf die Synovialmembran und verursacht heftige Schmerzen $[10,15]$. Bei Eröffnung der Sehnenscheide kann hingegen die Synovialflüssigkeit ablaufen, sodass der schmerzauslösende Druck nicht aufgebaut werden kann. In diesen Fällen ist die Lahmheit trotz septischer Infektion weniger deutlich als erwartet.

Die Lavage im Rahmen einer Tendovaginoskopie unter Vollnarkose hat verschiedene Vorteile:

- die Strukturen innerhalb der Sehnenscheide können visualisiert, beurteilt und falls nötig behandelt werden

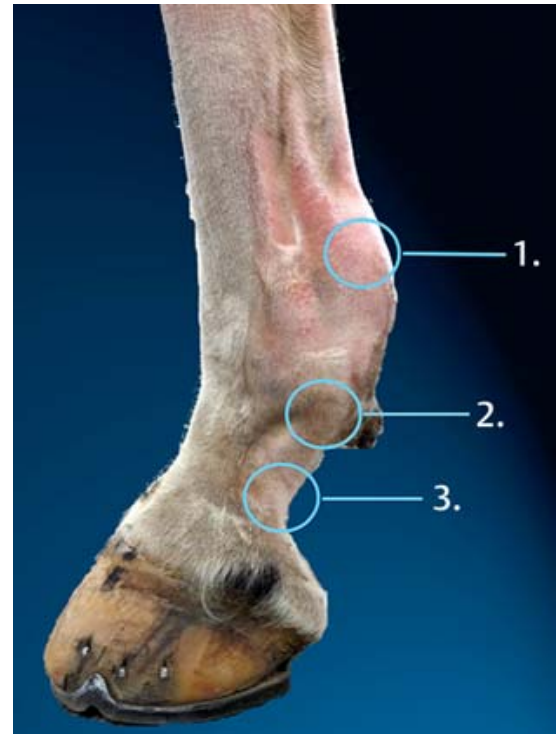

Abb. 3 Zugänge zur Fesselbeugesehnenscheide. Die Injektion erfolgt am aufgehobenen Bein. 1. Proximale Aussackung 2. Proximale Seitenblindsäcke an der Basis der Gleichbeine 3. Distale Aussackung palmar oder plantar zwischen der vierzipfligen Fesselplatte und der Sohlenbinde. (c) C. Gerdes

- eventuell vorhandene Fremdkörper können entfernt werden

- es kann mit einem größeren Volumen Flüssigkeit gespült werden

\section{Aseptische Tendovaginitis}

Das klinische Bild einer aseptischen Tendovaginitis ist unspezifisch und zeichnet sich meist durch folgende Symptome aus [18]:

- vermehrte Füllung der Sehnenscheide $(\triangleright$ Abb. 2)

- positive Beugeprobe des Fesselgelenks

- gering- bis mittelgradige Lahmheit

Bei der primären aseptischen Tendovaginitis sind keine pathologischen Veränderungen der Strukturen innerhalb der Sehnenscheide vorhanden. Trotzt sorgfältiger Diagnostik (Ultraschall, Röntgen mit und ohne Kontrastmittel) ist es häufig sehr schwierig, diese Veränderungen sicher auszuschließen [18]. Die genaue Überprüfung und Hinterfragung der Diagnose ist daher wichtig. Die Therapie erfolgt konservativ und besteht in folgenden Maßnahmen:

- Boxenruhe in Kombination mit kontrollierter Bewegung

- systemische Verabreichung nicht-steroidaler Antiphlogistika (NSAIDs)

> intrasynoviale Medikation 

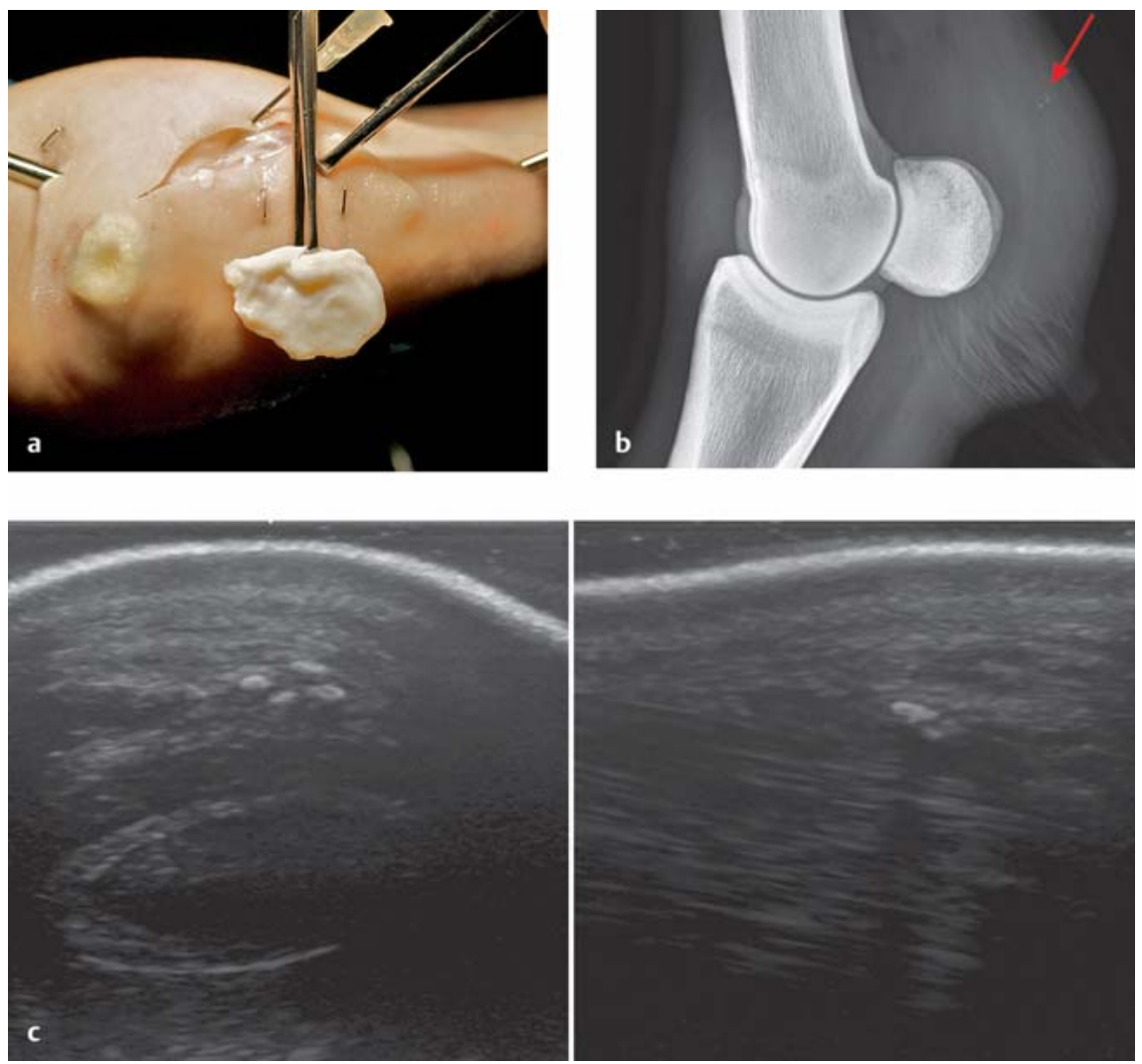

Abb. 4 a Intraoperative Aufnahme während Tendovaginoskopie der Fesselbeugesehnenscheide unter Vollnarkose nach Desmotomie des Fesselringbandes bei dystrophischer Mineralisierung. b Röntgenaufnahme des Fesselgelenks des Pferdes in $\mathbf{A b b}$. $\mathbf{4 a}$. Der rote Pfeil zeigt multifokale Verschattungen im Bereich des Fesselringbandes. c Transversales (links) und longitudinales (rechts) Ultraschallbild Zone 3C des Pferdes in - Abb. $\mathbf{4}$ a und b. Hyperechogene Bereiche im Fesselringband, die einen für Mineralisierung typischen „drop out“-Artefakt im Gewebe unterhalb des Befundes verursachen. (c) M. Head

- Aquatherapie/Cryotherapie (als initiale physikalische Therapie bei akuten Fällen)

- topische Medikation (Ziel: Entzündungshemmung und Hyperämisierung)

- Kompression mit Bandagen oder Verbänden

Zur intrasynovialen Medikation werden vorwiegend Glukokortikoide (Triamcinolon 5-10 mg oder Methylprednisolon Acetat 40-80 mg) injiziert, wahlweise in Kombination mit Hyaluronsäure zur Reduktion von Adhäsionen [11]. Die Maximaldosis von $18 \mathrm{mg}$ Triamcinolon und $200 \mathrm{mg}$ Methylprednisolon Acetat pro Pferd sollte nicht überschritten werden, um das Risiko einer iatrogenen Hufrehe als unerwünschte Nebenwirkung zu minimieren [7,12]. Glukokortikoide haben durch katabole Effekte einen negativen Einfluss auf die Heilung von Sehnen- und Bandschäden. Daher sollten sie nur angewendet werden, wenn keine Sehnen- oder Bandschäden vorliegen. Zur Injektion der Fesselbeugesehnenscheide gibt es - wie bei der lokalen Anästhesie - verschiedene Punktionsstellen ( $\mathrm{Abb}$. 3):

- lateraler Aspekt der proximalen Aussackung

- proximale Seitenblindsäcke an der Basis der Gleichbeine

- distale Aussackung (palmar oder plantar), zwischen der vierzipfeligen Fesselplatte und der Sohlenbinde

Topische Substanzen wie z.B. Dexamethason in DMSO oder Tensolvet ${ }^{\circledR}$ und Compagel $^{\circledR}$ (Heparin, Levomentor, Salicylat) werden häufig angewendet. Leider gibt es keine wissenschaftlichen Studien, die ihren Effekt bei der Behandlung von primärer Tendovaginitis der Fesselbeugesehnenscheide beweisen. Topisch aufgebrachte Salben sollten hyperämisierende und entzündungshemmende Bestandteile enthalten.
Eine sekundäre aseptische Tendovaginitis ist eine Sehnenscheidenentzündung, die durch pathologische Veränderungen der Strukturen innerhalb der Sehnenscheide oder der angrenzenden Strukturen verursacht wird. Aufgrund der Vielzahl möglicher Ursachen sollte von Fall zu Fall entschieden werden, ob die Therapie konservativ oder chirurgisch erfolgen sollte. Häufig ist eine Endoskopie der Sehnenscheide notwendig, um eine sichere Diagnose stellen zu können.

\section{Mögliche Befunde bei einer sekun- dären aseptischen Tendovaginitis}

Bei einer sekundären aseptischen Tendovaginitis können folgende Befunde erhoben werden, die entweder einzeln oder in komplizierten Fällen auch in Kombination auftreten können [18]:

- Desmitis des Fesselringbandes (PAL = Palmar/Plantar Annular Ligament) und Fesselringbandsyndrom [6]

- longitudinale Läsionen der tiefen Beugesehne [21]

- Tendinitis der oberflächlichen Beugesehne [16]

- Tendinitis der tiefen Beugesehne [3]

- Ruptur der Manica flexoria [8,9]

- Desmitis der distalen Sesambeinbänder

- Ruptur der Sehnenscheide [18]

- synoviale Ganglionzysten [4]

- Desmitis des Ligamentum intersesamoideum

Bei der Desmitis des Fesselringbandes kommt es zu einer Verdickung der Struktur und damit häufig auch zu einer Striktur des Fesselkanals (primäres Fesselringbandsyndrom). Eine sekundäre Striktur liegt vor, wenn eine oder mehrere Strukturen innerhalb des Fesselkanals eine Verengung verursachen. Dabei muss nicht immer eine Entzündung des Fesselringbandes vorliegen. Das Fesselringband setzt an den abaxialen Flächen der Gleichbeine an, verläuft palmar/plantar der Sehnenscheide direkt anliegend und bildet zusammen mit dem Lig. intersesamoideum einen Kanal, durch den die oberflächliche und die tiefe Beugesehnen laufen. Die physiologische Aufgabe des Fesselringbandes ist es, während der Standbein-Phase im Fesselgelenk den Zug auf die proximalen Sesambeine und die daran ansetzenden Schenkel des Musculus interosseus auszugleichen [5]. Durch die Ver- 
dickung des Fesselringbandes kommt es zu einer Beschränkung des freien Gleitens der Beugesehnen im Fesselkanal.

Die Ätiologie des Fesselringbandsyndroms ist häufig multifaktoriell:

- chronische Entzündung der Fesselbeugesehnenscheide

- externes Trauma

- Schwellung der Sehnen innerhalb der Sehnenscheide

- Überdehnung des Bandes bei der Extension des Fesselgelenks bei hohen Geschwindigkeiten

In chronischen Fällen kommt häufig eine fibrotische Verdickung des subkutanen Gewebes vor. Seltener werden Enthesiophyten an der Insertionsstelle der Gleichbeine festgestellt. Sonografisch ist das Band eine sehr dünne Struktur (max. 1$2 \mathrm{~mm}$ ), die nicht immer einfach darzustellen ist. Um festzustellen, ob eine Striktur des Fesselkanals vorliegt, kann der Raum zwischen der oberflächlichen Beugesehne und dem Fesselringband oder der Synovialmembran der Sehnenscheide sonografisch untersucht werden. Ist in diesem Raum Synovialflüssigkeit sichtbar, liegt keine Striktur vor. Dabei sollte der Schallkopf mit nur minimalem Druck aufgesetzt werden, um die Darstellung der vorhandenen Synovialflüssigkeit nicht zu verhindern.

Die Therapie ist in chronischen Fällen chirurgisch und besteht in einer Desmotomie des Fesselringbandes. Ob eine Desmotomie nötig ist, wird von Fall zu Fall entschieden. In normalen Sehnenscheiden sollte bei der Tendovaginoskopie ausreichend Raum für die Einführung der Instrumente in den Fesselkanal vorhanden sein. Ist dies nicht der Fall, liegt eine Striktur vor und eine Desmotomie des Fesselringbandes ist angezeigt. In einigen Fällen mit dystrophischer Mineralisierung ( Abb.4), Fremdkörpern oder synovialen Ganglionzysten kann eine komplette oder partielle Resektion des Fesselringbandes durchgeführt werden. Bei akuter, primärer Desmitis des Fesselringbandes ist initial ein konservativer Behandlungsversuch sinnvoll.

Longitudinale Läsionen der tiefen Beugesehne sind ein sehr häufiger Befund bei sekundärer chronischer Tendovaginitis [1,
18]. Allgemeine Tendinitiden der tiefen oder oberflächlichen Beugesehne werden in einer Vielzahl der Fälle festgestellt. Trotz ausführlicher Ultraschalluntersuchung und Röntgenuntersuchung mit Kontrastmittel ist es nicht einfach, eine sichere präoperative Diagnose zu stellen. Am schwierigsten darzustellen sind die lateralen Läsionen der tiefen Beugesehne. Sonografisch können die Läsionen in der Mehrzahl der Fälle nicht visualisiert werden. Befunde wie synoviale Hyperplasie und Fibrinablagerung im Bereich der Beugesehnen sind unspezifisch. Die Durchführung einer Tendovaginoskopie ist wichtig, um eine sichere Diagnose zu treffen.
Die Läsionen der tiefen Beugesehne haben eine deutlich schlechtere Prognose als andere mögliche Ursachen einer chronischen Tendovaginitis. Deswegen ist es wichtig, schnellstmöglich eine akkurate Diagnose zu stellen.

Die Läsionen können dann debridiert werden, um die bestmöglichen Voraussetzungen für eine Heilung zu schaffen. Die Besitzer von Pferden mit Sehnenschäden im Bereich der Fesselbeugesehnenscheide müssen mit einer Ruhezeit von 6-12 Monaten und häufigen Rezidiven rechnen. In einer Studie von Arensburg et al. [1] kehrten nur 38\% der Pferde mit einer longitu- 
dinalen Läsion der tiefen Beugesehne zu ihrem ursprünglichen Leistungsniveau zurück.

Im direkten Vergleich dazu haben Pferde mit einem Riss der Manica flexoria eine deutlich bessere Prognose. Nach chirurgischer Behandlung (Resektion oder Debridement der Manica flexoria) kehren 79\% zu ihrem ursprünglichen Leistungsniveau zurück [8]. Allerdings ist auch hier eine sichere präoperative Diagnosestellung allein sonografisch meist nicht möglich. Eine zusätzliche Röntgenkontrastmittelaufnahme der Fesselbeugesehnenscheide sollte daher routinemäßig zur Beurteilung der Manica flexoria durchgeführt werden [9].

Eine Desmitis der distalen Sesambeinbänder wird selten als alleiniger Befund bei sekundärer Tendovaginitis festgestellt. Häufiger treten diese Veränderungen sekundär und zusammen mit anderen Befunden auf. Die Untersuchung der Bänder erfolgt sonografisch an den Ansatzstellen am distalen Aspekt der Gleichbeine sowie am Fesselbein. Röntgenologisch sollte auf Enthesiophytenbildung untersucht werden.

\section{Fazit}

Die korrekte Diagnosestellung bei Lahmheiten, deren Ursache auf den Bereich der Fesselbeugesehnenscheide lokalisiert ist, ist sehr komplex. Es besteht eine Vielzahl an Differenzialdiagnosen und die zur Verfügung stehenden bildgebenden Verfahren sind nicht immer zuverlässig. Sonografisch können nur 49\% der endoskopisch diagnostizierten Läsionen korrekt festgestellt werden [18]. Um eine korrekte Diagnose zu stellen, ist in vielen Fällen die Durchführung einer Tendovaginoskopie der Fesselbeugesehnenscheide unerlässlich.

\section{Literatur}

1 Arensburg L, Wilderjans $H$, Simon $O$ et al. Nonseptic tenosynovitis of the digital flexor tendon sheath caused by longitudinal tears in the digital flexor tendons: A retrospective study of 135 tenoscopic procedures. Equine Vet J 2011; 43 (6): 660-668

2 Bailey SR, Elliott J. The corticosteroid laminitis story: 2. Science of if, when and how. Equine Vet J 2007; 39(1): 7-11

3 Barr AR, Dyson SJ, Barr FJ, O'Brien JK. Tendonitis of the deep digital flexor tendon in the distal metacarpal/metatarsal region associated with tenosynovitis of the digital sheath in the horse. Equine Vet J 1995; 27(5): 348-355

4 Crawford A, O'Donnell M, Crowe O et al. Digital Sheath Synovial Ganglion Cysts in Horses. Vet Surg 2011; 40(1): 66-72

5 Denoix JM. Functional anatomy of tendons and ligaments in the distal limbs (manus and pes). Vet Clin North Am Equine Pract 1994; 10(2): 273-322

6 Dik KJ, van den Belt AJ, Keg PR. Ultrasonographic evaluation of fetlock anular ligament constriction in the horse. Equine Vet J 1991; 23(4): 285288

7 Eustace RA, Redden RR. latrogenic laminitis. Vet Rec 1990; 126(23): 586

8 Findley JA, De Oliveira F, Bladon B. Tenoscopic Surgical Treatment of Tears of the Manica Flexoria in 53 horses. Vet Surg 2012; 41(8): 924-930

9 Fiske-Jackson AR, Barker WH, Eliashar E et al. The use of intrathecal analgesia and contrast radiography as preoperative diagnostic methods for digital flexor tendon sheath pathology. Equine Vet J 2013; 45(1): 36-40

10 Frisbie DD. Synovial joint anatomy, biology and pathobiology. In: Auer JA, ed. Equine Surgery. 4th ed. Philadelphia: WB Saunders; 2012: 1096-1114

11 Gaughan EM, Nixon AJ, Krook LP et al. Effect of sodium hylaluronate on tendon healing and adhesion formation in horses. Am J Vet Res 1991; 52(5): 764-773

12 Genovese RL. The use of corticosteroids in racetrack practice. In: Proceedings of the Symposium on Effective use of Corticosteroids in Veterinary Practice 1983; 56-65

13 Harper J, Schumacher J, Degraves F et al. Effects of analgesia of the digital flexor tendon sheath on pain originating in the sole, distal interphalan- geal joint or navicular bursa of horses. Equine Vet J 2007; 39(6): 535-539

14 Jordana M, Martens A, Duchateau L et al. Distal limb desensitisation following analgesia of the digital flexor tendon sheath in horses using four different techniques. Equine Vet J 2014; 46(4): 488-493

15 McDougall JJ. Arthritis and pain: Neurogenic origin of joint pain. Arthritis Res Ther 2006; 8(6): 220

16 Mcllwraith CW. Diseases of joints, tendons, ligaments and related structures. In: Stashak, ed. Adams' Lameness in Horses. 5. ed. Philadelphia: Lippincott Williams \& Wilkins; 2002: 459-644

17 Sampson SN, Schneider RK, Tucker RL et al. Magnetic resonance imaging feaures of oblique and straight distal sesamoidean desmitis in 27 horses. Vet Radiol Ultrasound 2007; 48(4): 303-311

18 Smith MR, Wright IM. Noninfected tenosynovitis of the digital flexor tendon sheath: a retrospective analysis of 76 cases. Equine Vet J 2006; 38 (2): 134-141

19 Smith LJ, Mellor DJ, Marr CM, Mair TS. What is the likelihood that a horse treated for septic digital tenosynovitis will return to its previous level of athletic function? Equine Vet J 2006; 38(4): 337-341

20 Wereszka MM, White NA 2nd, Furr MO. Factors associated with outcome following treatment of horses with septic tenosynovitis: 51 cases (1986-2003). J Am Vet Med Assoc 2007; 230 (8): 1195-1200

21 Wright IM, McMahon PJ. Tenosynovitis associated with longitudinal tears of the digital flexor tendons in horses: a report of 20 cases. Equine Vet J 1999; 31(1): 12-18

\section{Online}

http://dx.doi.org/10.1055/s-0034-1396298

Carolin Gerdes, MRCVS

Rossdale \& Partners

Cotton End Road

CB8 7HL Exning, Newmarket

UK 